Mathematical Modelling and Analysis

Volume 5, 2000, PAGes 127-132

(C) 2000 Technika

\title{
SOME ESTIMATES OF SPECIAL CLASSES OF INTEGRALS
}

\author{
T.I. MALYUTINA ${ }^{1}$ \\ Ukrainian Academy of Banking \\ PetroPavlovska Str. 56, Sumy, 244030, Ukraine \\ E-mail: malyutin@academia.sumy.ua
}

Received July 1, 1999

\begin{abstract}
We study the integrals $\int_{a}^{b} f(t) \exp \left(i|\ln r t|^{\sigma}\right) d t$ and obtain asymptotic formula for these functions of non-regular growth. This is a peculiar kind of the theory asymptotic expansions. In particular, we get asymptotic formulae for different entire functions of non-regular growth. Asymptotic formulas for Levin-Pfluger entire functions of completely regular growth are well-known [1]. Our formulas allow to find limiting Azarin's [2] sets for some subharmonic functions. The kernel $\exp \left(i|\ln r t|^{\sigma}\right)$ contains arbitrary parameter $\sigma>0$. The integrals for $\sigma \in(0,1), \sigma=1, \sigma>1$ essentially differ. Our arguments can apply to more general kernels. We give a new variant of the classic lemma of Riemann and Lebesgue from the theory of the transformation of Fourier.
\end{abstract}

\section{THE ANALOGY OF RIEMANN'S-LEBESGUE'S LEMMA}

We will begin with the analogy of Riemann's-Lebesgue's lemma.

Lemma 1.1. Let $f(t) \in \mathbf{L}_{1}([a, b]), 0 \leq a<b \leq \infty, \sigma>1$. Then

$$
\lim _{r \rightarrow \infty} \int_{a}^{b} f(t) \exp \left(i|\ln r t|^{\sigma}\right) d t=0 .
$$

Proof. Assume that $a>0, f \in \mathbf{C}_{\mathbf{1}}([a, b])$. Integrating by parts, we get

$$
\int_{a}^{b} f(t) \exp \left(i|\ln r t|^{\sigma}\right) d t=\left.\frac{f(t) t \exp \left(i(\ln r t)^{\sigma}\right)}{i \sigma(\ln r t)^{\sigma-1}}\right|_{a} ^{b}-
$$

\footnotetext{
${ }^{1}$ The author expresses gratitude to A.F. Grishin for valuable remarks made in the process of this work.
} 


$$
\frac{1}{i \sigma} \int_{a}^{b}\left[\frac{f^{\prime}(t) t+f(t)}{(\ln r t)^{\sigma-1}}-\frac{(\sigma-1) f(t) t}{(\ln r t)^{\sigma}}\right] \exp \left(i(\ln r t)^{\sigma}\right) d t .
$$

Limit of right part equals zero if $r \rightarrow \infty$.

Now let $f \in \mathbf{L}_{\mathbf{1}}([a, b])$ and let function $f_{1} \in \mathbf{C}_{\mathbf{1}}([a, b])$ such that

$$
\int_{a}^{b}\left|f(t)-f_{1}(t)\right| d t \leq \epsilon
$$

where $\epsilon$ is any positive number. Then,

$$
\left|\int_{a}^{b} f(t) \exp \left(i(\ln r t)^{\sigma}\right) d t\right| \leq\left|\int_{a}^{b} f_{1}(t) \exp \left(i(\ln r t)^{\sigma}\right) d t\right|+\epsilon
$$

The desirable conclusion follows from above proved.

Let $a=0, f \in \mathbf{L}_{\mathbf{1}}([0, b])$, and let $\epsilon>0$ be on arbitrary number. If $\left|\exp \left(i|\ln r t|^{\sigma}\right)\right| \leq 1$, then there exists constants $\delta>0$ such that

$$
\left|\int_{0}^{\delta} f(t) d t\right| \leq \epsilon
$$

Then

$$
\left|\int_{0}^{b} f(t) \exp \left(i|\ln r t|^{\sigma}\right) d t\right|=\left|\int_{0}^{\delta}+\int_{\delta}^{b}\right| \leq \epsilon+\left|\int_{\delta}^{b} f(t) \exp \left(i|\ln r t|^{\sigma}\right) d t\right| .
$$

Remark 1.1. Lemma is true if kernels $\exp \left(i|\ln r t|^{\sigma}\right)$ are replaced by $\exp (i \varphi(r t) \ln r t)$, where $\varphi$ is differentialable increasing function on the half axis $[0, \infty)$ such that $\lim _{t \rightarrow \infty} \varphi(t)=\infty$.

We do not evaluate the speed of convergence to zero of the integral. It can't be done $f \in \mathbf{L}_{1}([a, b])$.

\section{AZARIN LIMITING SETS}

In this section we consider the following function:

$$
\begin{aligned}
u_{1}(z, \sigma)= & \frac{r \sin \theta}{\pi} \int_{0}^{\infty} \frac{\tau^{\rho} \exp \left(i \lambda|\ln \tau|^{\sigma}\right)}{\tau^{2}-2 \tau r \cos \theta+r^{2}} d \tau= \\
& \frac{r^{\rho} \sin \theta}{\pi} \int_{0}^{\infty} \frac{t^{\rho} \exp \left(i \lambda|\ln t r|^{\sigma}\right)}{t^{2}-2 t \cos \theta+1} d t
\end{aligned}
$$




$$
\begin{aligned}
u_{2}(z, \sigma)= & \frac{1}{\pi} \int_{0}^{\infty} \frac{r(r-\tau \cos \theta)}{\tau^{2}-2 \tau r \cos \theta+r^{2}} \exp \left(i \lambda|\ln \tau|^{\sigma}\right) d \tau= \\
& \frac{r^{\rho}}{\pi} \int_{0}^{\infty} \frac{1-t \cos \theta}{t^{2}-2 t \cos \theta+1} \exp \left(i \lambda|\ln t r|^{\sigma}\right) d t
\end{aligned}
$$

$u_{3}(z, \sigma)=\operatorname{Re} u_{1}(z, \sigma), u_{4}(z, \sigma)=\operatorname{Im} u_{1}(z, \sigma), u_{5}(z, \sigma)=\operatorname{Re} u_{2}(z, \sigma)$,

$u_{6}(z, \sigma)=\operatorname{Im} u_{2}(z, \sigma), z=r e^{i \theta}, \rho \in(0,1), \sigma>0, \lambda \geq 0$. If $\sigma=1$, we do not write module.

Azarin limiting set $F r u$ of subharmonic function $u(z)$ is its significant characteristics of the growth [2]. Fru is limiting set of the family of functions $u_{t}(z)=u(t z) / t^{\rho}$ ( $\rho$ be the order of $u$ ) by $t \rightarrow+\infty$ in the topology of the space of generalized Shwartz's functions. If $\rho \in(0,1), \sigma \in(0,1), \lambda>0$ we have the following properties:

$$
\begin{aligned}
& \operatorname{Fr} u_{3}=\operatorname{Fr} u_{4}=\left\{\alpha \frac{\sin \rho(\pi-\theta)}{\sin \rho \pi} r^{\rho}: \alpha \in[-1,1]\right\}, \\
& \operatorname{Fr} u_{5}=\operatorname{Fr} u_{6}=\left\{\alpha \frac{\cos \rho(\pi-\theta)}{\sin \rho \pi} r^{\rho}: \alpha \in[-1,1]\right\} .
\end{aligned}
$$

Let $h_{k}(\theta)$ be the Fragmen-Lindeljeff indicator of the function $u_{k}(z, \sigma)$. Then the following relations hold:

$$
h_{3}(\theta)=h_{4}(\theta)=\frac{|\sin \rho(\pi-\theta)|}{\sin \rho \pi}, \quad h_{5}(\theta)=h_{6}(\theta)=\frac{|\cos \rho(\pi-\theta)|}{\sin \rho \pi} .
$$

Theorem 2.1. Let $\sigma=1$, and let $\rho \in(0,1), \lambda \geq 0$ be given numbers. Then the following relations hold:

$$
\begin{aligned}
& u_{3}(z, \sigma)=\left[A_{\rho}(\lambda, \theta) \cos \lambda \ln r-B_{\rho}(\lambda, \theta) \sin \lambda \ln r\right] r^{\rho}, \\
& u_{4}(z, \sigma)=\left[B_{\rho}(\lambda, \theta) \cos \lambda \ln r+A_{\rho}(\lambda, \theta) \sin \lambda \ln r\right] r^{\rho}, \\
& u_{5}(z, \sigma)=\left[C_{\rho}(\lambda, \theta) \cos \lambda \ln r-D_{\rho}(\lambda, \theta) \sin \lambda \ln r\right] r^{\rho}, \\
& u_{6}(z, \sigma)=\left[D_{\rho}(\lambda, \theta) \cos \lambda \ln r+C_{\rho}(\lambda, \theta) \sin \lambda \ln r\right] r^{\rho},
\end{aligned}
$$

where

$$
A_{\rho}(\lambda, \theta)=\operatorname{Re} \frac{\sin (\rho+i \lambda)(\pi-\theta)}{\sin (\rho+i \lambda) \pi}, \quad B_{\rho}(\lambda, \theta)=\operatorname{Im} \frac{\sin (\rho+i \lambda)(\pi-\theta)}{\sin (\rho+i \lambda) \pi} .
$$

Analogous formulae for $C_{\rho}(\lambda, \theta)$ and $D_{\rho}(\lambda, \theta)$ come out if $\sin (\rho+i \lambda)(\pi-\theta)$ is replaced by $\cos (\rho+i \lambda)(\pi-\theta)$.

Corollary 2.1.

$$
\begin{aligned}
& \text { Fr } u_{5}(z, 1)=\left\{C_{\rho}(\lambda, \theta) \sin \varphi-D_{\rho}(\lambda, \theta) \cos \varphi: \varphi \in[0,2 \pi]\right\}, \\
& h_{5}(\theta)=\sqrt{C_{\rho}^{2}(\lambda, \theta)+D_{\rho}^{2}(\lambda, \theta)},
\end{aligned}
$$


and analogous formulae for $u_{3}(z, 1), u_{4}(z, 1), u_{6}(z, 1)$ occur.

Proof. We prove equality (2.4). We have

$$
u_{3}(z, 1)=\frac{r^{\rho} \sin \theta}{\pi} \Re r^{i \lambda} \int_{0}^{\infty} \frac{t^{\rho+i \lambda}}{t^{2}-2 t \cos \theta+1} d t .
$$

We define the digit branch of function $t^{\rho+i \lambda}$ on cut plane by the semi-axis $[0, \infty)$ such that $\arg t=0$ over the side of the cut, $\arg t=2 \pi$ under the side of the cut, and $0<\arg t<2 \pi$ on the plane with the cut.

We define the contour of integration $L=L(\epsilon) \bigcup L(R) \bigcup L_{1} \bigcup L_{2}$, where $L(\epsilon)=\{z:|z| \leq \epsilon\}, L(R)$ is analogous circle with the radius $R, L_{1}$ is upper side of the cut of $[\epsilon, R], L_{2}$ is the bottom of this cut with has contrary respect. Then we have

$$
\begin{gathered}
I=\int_{L} \frac{t^{\rho+i \lambda}}{t^{2}-2 t \cos \theta+1} d t=I(\epsilon)+I(R)+I_{1}+I_{2}, \\
\lim _{\epsilon \rightarrow 0} I(\epsilon)=\lim _{R \rightarrow \infty} I(R)=0,
\end{gathered}
$$

and

$$
\begin{gathered}
I_{1}=\int_{\epsilon}^{R} \frac{t^{\rho+i \lambda}}{t^{2}-2 t \cos \theta+1} d t \\
I_{2}=\int_{R}^{\epsilon} \frac{\exp (2 \pi i(\rho+i \lambda)) t^{\rho+i \lambda}}{t^{2}-2 t \cos \theta+1} d t=-\exp (2 \pi i(\rho+i \lambda)) I_{1} .
\end{gathered}
$$

The integral function has simple poles at points $t_{1}=\exp (i \theta)$ and $t_{2}=\exp (i(2 \pi-\theta))$ if $\theta \neq 0$. Applying the residue theorem, we have

$$
\begin{gathered}
I=2 \pi i\left(\operatorname{Res}_{t=e^{i \theta}} \frac{t^{\rho+i \lambda}}{t^{2}-2 t \cos \theta+1}+\operatorname{Res}_{t=e^{i(2 \pi-\theta)}} \frac{t^{\rho+i \lambda}}{t^{2}-2 t \cos \theta+1}\right)= \\
\pi i\left(\frac{e^{i \theta(\rho+i \lambda)}}{e^{i \theta}-\cos \theta}+\frac{e^{i(2 \pi-\theta)(\rho+i \lambda)}}{e^{i(2 \pi-\theta)}-\cos \theta}\right)=\frac{\pi}{\sin \theta}\left(e^{i \theta(\rho+i \lambda)}-e^{i(2 \pi-\theta)(\rho+i \lambda)}\right)= \\
\frac{\pi}{\sin \theta}[\cos \theta(\rho+i \lambda)+i \sin \theta(\rho+i \lambda)-\cos (2 \pi-\theta)(\rho+i \lambda)-i \sin (2 \pi-\theta)(\rho+i \lambda)] \\
=\frac{\pi}{\sin \theta}[2 \sin (\pi-\theta)(\rho+i \lambda) \sin \pi(\rho+i \lambda)-2 i \sin (\pi-\theta) \cos \pi(\rho+i \lambda)]= \\
\frac{2 \pi}{\sin \theta} \sin (\pi-\theta)(\rho+i \lambda)[\sin \pi(\rho+i \lambda)-i \cos \pi(\rho+i \lambda)] .
\end{gathered}
$$

Equality (2.11) implies

$$
I_{1}+I_{2}=(1-\exp (2 \pi(\rho+i \lambda))) I_{1}=(1-\cos 2 \pi(\rho+i \lambda)-
$$




$$
i \sin 2 \pi(\rho+i \lambda)) I_{1}=2 \sin \pi(\rho+i \lambda)[\sin \pi(\rho+i \lambda)-i \cos \pi(\rho+i \lambda)] I_{1} .
$$

This and (2.10) give

$$
\int_{0}^{\infty} \frac{t^{\rho+i \lambda}}{t^{2}-2 t \cos \theta+1} d t=\frac{\pi}{\sin \theta} \frac{\sin (\pi-\theta)(\rho+i \lambda)}{\sin \pi(\rho+i \lambda)} .
$$

This implies assertion (2.4) of the theorem. If $\theta=0$ then (2.4) can be received by $\theta \rightarrow+0(2 \pi-0)$.

\section{ASYMPTOTIC FORMULAE OF INTEGER FUNCTIONS OF IRREGULAR GROWTH}

Let $\left\{a_{k}\right\}_{k-1}^{\infty}$ be a sequence of positive zeros of the integer function $f(z)$, and let $\rho \in(0,1)$ be the order of $f$. Define $\ln \left(1-z / a_{k}\right)$ by $\ln \left(1-z / a_{k}\right)>0$, if $z \in(-\infty, 0)$, on the cut plane by $[0, \infty)$. Then we have

$$
\ln f(z)=\sum_{k=1}^{\infty} \ln \left(1-\frac{z}{a_{k}}\right)=\int_{0}^{\infty} \ln \left(1-\frac{z}{t}\right) d n(t)=\int_{0}^{\infty} \frac{z}{z-t} \frac{n(t)}{t} d t
$$

where $n(t)$ is defined to be the number of zeros, counted with multiplicity, of $f$ in the circle of radius $t$, excluding those at the origin. We have

$$
\ln |f(z)|=\int_{0}^{\infty} \frac{r(r-t \cos \theta)}{t^{2}-2 t r \cos \theta+r^{2}} \frac{n(t)}{t} d t .
$$

Define $\varphi(t)$ by $\varphi(t)=t^{\rho}\left(a_{0}+a_{1} \cos \lambda \ln t+b_{1} \sin \lambda \ln t\right)$.

If $a_{0} \geq \sqrt{1+\lambda^{2} / \rho^{2}} i \sqrt{a_{1}^{2}+b_{1}^{2}}$, then $\varphi(t)$ is the increasing function so as

$$
\varphi^{\prime}(t)=\rho t^{\rho-1}\left[a_{0}+\cos \lambda \ln t\left(a_{1}+\frac{\lambda}{\rho} b_{1}\right)+\sin \lambda \ln t\left(b_{1}-\frac{\lambda}{\rho} a_{1}\right)\right] \geq 0 .
$$

Consider the function $f$ with $n(t)=[\varphi(t)]$ (here [.] represents the integer part). Azarin limiting set $F r f$ of the integer function $f$ is Azarin limiting set of the subharmonic function $\ln |f(z)|$. Applying the theorem 2.1, we obtain

$$
\begin{gathered}
\operatorname{Fr} f=\left\{\left(a_{0} \frac{\cos \rho(\pi-\theta)}{\sin \rho \pi}+\left(a_{1} C_{\rho}(\lambda, \theta)+b_{1} D_{\rho}(\lambda, \theta)\right) \cos \varphi+\right.\right. \\
\left.\left.\left(-a_{1} D_{\rho}(\lambda, \theta)+b_{1} C_{\rho}(\lambda, \theta)\right) \sin \varphi\right) r^{\rho}: \varphi \in[0,2 \pi]\right\}, \\
h_{f}(\theta)=a_{0} \frac{\cos \rho(\pi-\theta)}{\sin \rho \pi}+\sqrt{a_{1}^{2}+b_{1}^{2}} \sqrt{C_{\rho}^{2}(\lambda, \theta)+D_{\rho}^{2}(\lambda, \theta)} .
\end{gathered}
$$


These relations hold if $a_{0}<\sqrt{1+\lambda^{2} / \rho^{2}} \sqrt{a_{1}^{2}+b_{1}^{2}}$. However, function $f$ will be meromorphic function in a general case. If

$$
\varphi(t)=t^{\rho}\left(a_{0}+\sum_{k=1}^{n}\left(a_{k} \cos \lambda_{k} \ln t+b_{k} \sin \lambda_{k} \ln t\right)\right)
$$

then using the theorem 2.1, we obtain asymptotic formulae for $\ln |f(z)|$. If $\varphi(t)$ is the increasing function then $f$ is the integer function. In this way, we can obtain asymptotic formulae for a general class of integer functions of irregular growth. In the book of B.Ya.Levin [1], asymptotic formulae for a class of integer functions of regular growth are represented.

\section{REFERENCES}

[1] B.Ja. Levin. Distributions of Zeros of Entire Functions. GITL, Moscow, 1956. (in Russian)

[2] V.S. Azarin. On Asymptotic Behavior of Subharmonic Functions. Mat. Sb., 108 (2), $1979,147-167$.

\section{SPECALIƯJU INTEGRALUૃ KLASIỤ IVERČIAI}

\section{T.I. MALIUTINA}

Darbe nagrinëjami integralai $\int_{a}^{b} f(t) \exp \left(i|\ln r|^{\sigma}\right) d t$ ir tiriamos šių nereguliaraus augimo greičiu funkciju asimptotinès formulès. Gautos naujos asimptotinès formulès, leidžiančios rasti Azarino aibes kai kurioms subharmoninėms funkcijoms. Branduolys $\exp \left(i|\ln r t|^{\sigma}\right)$ priklauso nuo vieno parametro $\sigma>0$. Trys atvejai, kai $0<\sigma<1, \sigma=1$ ir $\sigma>0$, yra esminiai skirtingi. Darbo metodika gali būti naudojama ir bendresniems branduoliu atvejams. İrodytas naujas Rimano ir Lebego lemos varijantas, kuris naudojamas Furje transformacijos teorijoje. 